\title{
Evaluation of Peak Reflux Velocities with Vector Flow Imaging and Spectral Doppler Ultrasound in Varicose Veins
}

\section{(c) (1) $\ominus$}

Authors

Thor Bechsgaard', Kristoffer Lindskov Hansen², Andreas Brandt², Ramin Moshavegh ${ }^{4}$, Julie Lyng Forman ${ }^{5}$, Pia Føgh $^{6}$, Lotte Klitfod $^{6}$, Niels Bækgaard ${ }^{6}$, Lars Lönn 7 , Jørgen Arendt Jensen, ${ }^{8}$ Michael Bachmann Nielsen ${ }^{1}$

Affiliations

1 Righhospitalet, Radiology, Copenhagen, Denmark

2 Copenhagen University Hospital, Department of Diagnostic Radiology, Copenhagen, Denmark

3 Copenhagen University Hospital Rigshospitalet, Department of Diagnostic Radiology, 2100 Copenhagen Oe, Denmark

4 The Technical University of Denmark, Department of Electrical Engineering Center for Fast Ultrasound Imaging, 2800 Kgs. Lyngby, Denmark

5 Copenhagen University, Department of Public Health Section of Biostatistics, 1014 Copenhagen K, Denmark

6 Copenhagen University Hospital Rigshospitalet, Department of Vascular Surgery Herlev \& Gentofte Hospital, 2900 Hellerup, Denmark

7 Rigshospitalet, Copenhagen, DK, Radiology, Copenhagen, Denmark

8 Technical University of Denmark, Department of Electrical Engineering Center for Fast Ultrasound Imaging, 2800 Kgs. Lyngby, Denmark

\section{Keywords}

ultrasound, ultrasound-spectral Doppler, veins,

peak velocity, vector flow imaging

received $\quad 15.12 .2017$

revised 24.04.2018

accepted 17.05.2018

Bibliography

DOI https://doi.org/10.1055/a-0643-4430

Ultrasound Int Open 2018; 4: E91-E98

(c) Georg Thieme Verlag KG Stuttgart · New York

ISSN 2199-7152
Correspondence

Mr. Thor Bechsgaard, MD

Rigshospitalet, Radiology

Blegdamsvej 9

2100, Copenhagen

Denmark

Tel.: +45/203/70 727

thorbechsgaard@gmail.com

\section{ABSTRACT}

Purpose Spectral Doppler ultrasound (SDUS) is used for quantifying reflux in lower extremity varicose veins. The technique is angle-dependent opposed to the new angle-independent Vector Flow Imaging (VFI) method. The aim of this study was to compare peak reflux velocities obtained with VFI and SDUS in patients with chronic venous disease, i.e., pathological retrograde blood flow caused by incompetent venous valves.

Materials and Methods 64 patients with chronic venous disease were scanned with VFI and SDUS in the great or the small saphenous vein, and reflux velocities were compared to three assessment tools for chronic venous disease. A flow rig was used to assess the accuracy and precision of the two methods. Results The mean peak reflux velocities differed significantly (VFI: $47.4 \mathrm{~cm} / \mathrm{s}$ vs. SDUS: $62.0 \mathrm{~cm} / \mathrm{s}, \mathrm{p}<0.001$ ). No difference in absolute precision $(p=0.18)$ nor relative precision $(p=0.79)$ was found. No correlation to disease severity, according to assessment tools, was found for peak reflux velocities obtained with either method. In vitro, VFI was more accurate but equally precise when compared to SDUS.

Conclusion Both VFI and SDUS detected the pathologic retrograde flow in varicose veins but measured different reflux velocities with equal precision. VFI may play a role in evaluating venous disease in the future.

\section{Introduction}

Ultrasound is used to evaluate venous diseases of the lower extremities [1-3]. Pathological retrograde blood flow, i. e. reflux, in the superficial veins of the lower extremities indicates chronic venous disease. B-mode ultrasound is used for anatomical mapping, color
Doppler ultrasound for detecting reflux, and spectral Doppler ultrasound (SDUS) for quantification of reflux duration and velocities [4]. Duplex ultrasound (B-mode and color Doppler ultrasound) is sufficient in patients presenting solely with varicose veins, while a more severe condition with additional findings, like edema and trophic skin changes due to chronic venous insufficiency, may war- 
rant a more detailed examination with triplex ultrasound (duplex and SDUS) or other imaging modalities, like computed tomography or invasive phlebography [1].

Chronic venous disease is commonly caused by valve incompetence in the great saphenous vein of the thigh or the small saphenous vein of the lower leg [1]. The pathophysiologic cause is in the superficial or the deep venous system, rarely in both [1]. Reflux velocity values measured with SDUS are correlated with worsening physical findings [4,5], and symptoms in chronic venous disease [6]. However, conventional SDUS is limited by angle dependency and due to the anatomical course of the superficial veins of the lower extremities, beam-steering is required to achieve acceptable insonation angles for peak reflux velocity estimations [7].

Vector Flow Imaging (VFI) is an angle-independent ultrasound technique [8] validated in various vessel geometries and examined for different cardiovascular diseases [9-19]. Recently, the blood flow velocities in the veins of the lower extremities have been evaluated with VFI in healthy volunteers with promising results [20,21]. The results of this study were exclusively obtained with the VFI technique implemented on a commercial ultrasound scanner from BK Ultrasound. However, other VFI techniques exist. Dunmire et al. summarized the studies with cross-beam vector Doppler ultrasound [22], while Jensen et al. recently summed up the technical approaches of vector flow imaging [23, 24]. Subsequently, Hansen et al. outlined several in vivo applications [25], Goddi et al. described the innovative perspectives of VFI [26], and Au et al. reviewed high-frame-rate VFI with plane wave emissions [27].

The aim of this study was to compare venous peak reflux velocity measurements obtained with VFI and SDUS in adults with chronic venous disease. The hypothesis was that VFI estimates peak reflux velocities with a precision similar to SDUS. A secondary aim was to correlate peak reflux velocities measured by VFI and SDUS with three assessment tools routinely used in the evaluation of chronic venous disease. Additionally, flow phantom measurements were performed to estimate the accuracy and precision of both methods in a controlled setup.

\section{Materials and Methods}

\section{Patients}

64 patients with varicose vein disease were prospectively included in a tertiary venous center from August 1, 2016 to January 31, 2017 after informed consent ( $\triangleright$ Table 1). The study was approved by the Danish National Committee on Biomedical Research Ethics and the local ethics committee ( $\mathrm{H}-15007065)$, as well as by the Danish Data Protection Agency (2012-58-0004).

\section{VFI}

The VFI technique is based on the transverse oscillation ultrasound method and can estimate blood flow velocities in an angle-independent manner [8]. In VFI, a conventional pulse for Doppler estimation is emitted, and by using a special estimator and changing the apodization in receive, the axial and transverse velocity components are found simultaneously. The blood flow is visualized in a color box, a color-wheel indicates the direction of the blood flow, and superimposed arrows in the color box ease the interpretation of the flow direction and magnitude ( $\triangleright$ Fig. 1).

\section{Data collection and data processing}

The VFI and SDUS recordings were obtained with the same commercial ultrasound scanner (BK3000, BK Ultrasound, Herlev, Denmark) and a 2-8 MHz linear transducer (8L2, BK Ultrasound, Herlev, Denmark). VFI peak velocities acquired from data files were exported and analyzed off-line with an algorithm developed in-house for MATLAB (MathWorks, Natick, MA, USA) after the patients had completed their participation in the research project. The SDUS peak velocities were acquired from screenshots of spectrograms and analyzed off-line in a professional quality vector graphics editor (Inkscape, C/O Software Freedom Conservancy, Brooklyn, NY, USA).

\section{Flow phantom}

In a flow phantom (Cole-Parmer centrifugal pump, Vernon Hills, IL, USA), velocities of 50 and $60 \mathrm{~cm} / \mathrm{s}$ were examined with VFI and SDUS to establish the accuracy and precision of the two techniques

- Table 1 Characteristics of study population (patients).

\section{Dichotomous variables}

No. of patients (sex in percent)

Symptomatic side

Clinical score in CEAP

Etiology in CEAP

Anatomy in CEAP

Pathophysiology in CEAP

Advanced CEAP
64 ( $67 \%$ females, $33 \%$ males)

31 right lower extremities, 33 left lower extremities

59 with varicose veins (C2), 3 with edema (C3), 2 with skin changes (C4)

63 with primary disease, 1 with secondary disease

63 with pathology in superficial veins, 1 with pathology in both the superficial and deep veins

63 with reflux and 1 with reflux and obstruction

59 with disease in the greater saphenous vein above the knee, 5 with disease in the lesser saphenous vein

\begin{tabular}{|l|c|c|}
\hline Continuous variables & Mean (range) & St. dev. \\
\hline Age & $56.98(29-90)$ & 16.23 \\
\hline BMI & $25.76(19-37)$ & 4.45 \\
\hline $\begin{array}{l}\text { Venous segmental } \\
\text { disease score }\end{array}$ & $1.04(1-3)$ & 0.27 \\
\hline Prior surgery & $0.55(0-5)$ & 0.97 \\
\hline Depth in mm & $9.38(2-20)$ & 4.29 \\
\hline Diameter in mm & $5.91(3-12)$ & 1.72 \\
\hline
\end{tabular}

Abbreviations: St. dev. $=$ standard deviation, $\mathrm{BMI}=$ body mass index, $\mathrm{CEAP}=$ Clinical-Etiology-Anatomy-Pathophysiology classification, $\mathrm{C2}=$ clinical - varicose veins, $C 3$ = clinical - edema, $C 4=$ clinical - skin changes 


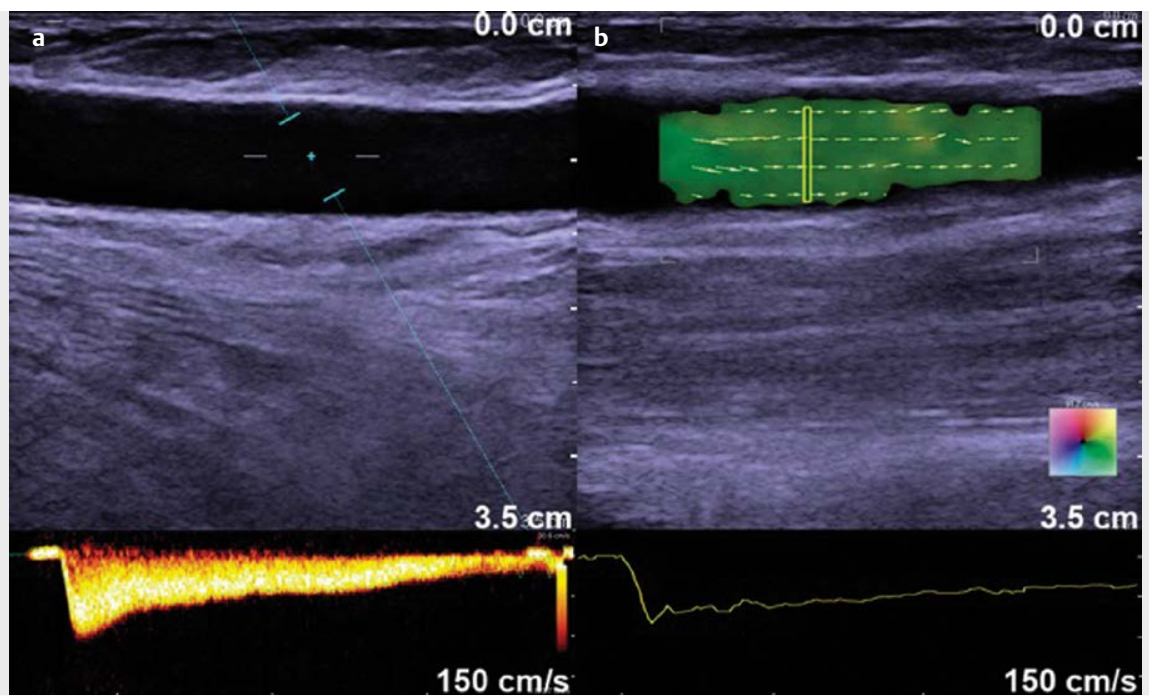

-Fig. 1 a Longitudinal SDUS scan of superficial lower extremity vein with valve incompetence causing reflux. The range gate covers the vessel without including vessel boundaries and $30^{\circ}$ beam steering is applied reducing the insonation angle from $90^{\circ}$ to $60^{\circ}$. The spectrogram for the SDUS data is shown at the bottom of the figure. $\mathbf{b}$ Corresponding longitudinal VFI scan. The VFI region of interest was placed corresponding to the position of the SDUS range gate. A 2D vector velocity map is displayed with VFI to depict the directions and magnitudes of the blood flow. A color wheel is provided and superimposed arrows on the color-coded pixels ease the velocity interpretation. Velocity curve for VFI data is shown at the bottom of the figure.

under controlled settings. The tested in vitro velocities were selected to correspond to the mean peak reflux velocity of the two techniques found in the in vivo study, where the mean in vivo peak reflux velocity was approx. $50 \mathrm{~cm} / \mathrm{s}$ for VFI and $60 \mathrm{~cm} / \mathrm{s}$ for SDUS. Ten replicate measurements were made with both methods at the two velocity settings. A constant flow was examined with the transducer at a fixed examination depth $(2.5 \mathrm{~cm})$ and insonation angle (VFI $90^{\circ}$ vs. SDUS $60^{\circ}$ due to $30^{\circ}$ beam steering). The reference velocities were measured by a magnetic flow meter (MAG1100, Danfoss, Nordborg, Denmark) with a stated accuracy of $\pm 1 \%$.

\section{The in vivo setup}

64 patients with chronic venous disease of the lower extremities underwent evaluation of peak reflux velocity estimations in the great or the small saphenous vein. A standardized approach was used to provoke the retrograde blood flow and to obtain the velocities [28]. For VFI, an insonation angle approximating $90^{\circ}$ was applied, while for SDUS, an insonation angle of $60^{\circ}$ was applied due to $30^{\circ}$ beam steering. There was no difference in the position of the transducer on the surface of the skin for corresponding estimations with the two methods, as the difference in insonation angle was solely due to the beam steering ( $\triangleright$ Fig. 1 ). The great saphenous vein was examined in the mid-to-distal-thigh region, and the small saphenous vein was examined in the proximal-to-mid-calf region.

A previously described setup for evaluating deep venous reflux [29], and reproduced for examination of insufficient great and small saphenous veins was used $[4,5,28]$. Each patient underwent evaluation of an insufficient vein of the symptomatic extremity. The patients were weight bearing on the opposite extremity than the one examined during the recordings to ensure that the leg examined with US remained relaxed during the examination. Reflux was provoked by the cuff compression-decompression system (Rapid Cuff Inflation System, Hokanson Bellevue, WA, USA) that inflated a 12-cm cuff to $80 \mathrm{~mm} \mathrm{Hg}$, which compressed the lower leg for $3 \mathrm{~s}$ prior to rapid decompression in $0.3 \mathrm{~s}$. During decompression, the peak reflux velocity was recorded. The recordings were replicated three times with 60 s intervals between recordings as suggested by others [30]. Between measurements, the transducer was lifted from the skin, and the settings were optimized. This procedure was performed for VFI as well as for SDUS. For both methods, the reported reflux velocity for each patient was an average estimate over the three replicated recordings. A similar setup has previously been described for evaluating antegrade venous blood flow velocities [21].

Manual angle correction by tilting the transducer was not performed to avoid influencing the blood flow velocity by incidentally compressing the examined vein. All recordings were performed by one medical doctor (TB), and optimal settings were set for each of the two techniques. The mean pulse repetition frequency was $5.09 \mathrm{kHz}$ for VFI and $5.08 \mathrm{kHz}$ for SDUS, both ranging from $3 \mathrm{kHz}$ to $7 \mathrm{kHz}$. For SDUS, the D-gain was set at $60 \%$, the D-frequency at $3.8 \mathrm{MHz}$, the B-frequency at $7.5 \mathrm{MHz}$ and the $\mathrm{B}$-gain at $50 \%$, while these specifications were not recorded for $\mathrm{VFI}$.

Two ultrasound artifacts were noticed during collection of VFI data, also known from conventional Doppler ultrasound, i. e. blooming and aliasing [31,32]. Blooming is random noise covering the vessel wall and surroundings due to incorrect adjustment of color gain. In the VFI examination, blooming artifacts were avoided by adjusting the vector gain to the highest setting possible, to fill the lumen with flow signal without noise from the surrounding soft tissue. Aliasing arises in pulsed Doppler systems when the Doppler shift of the moving blood scatterer is more than half the pulse repetition frequency (PRF) corresponding to the Nyquist limit [33]. Flow direction and velocity will be displayed reversed due to the wrap-around effect. When aliasing artifacts were encountered during the VFI reflux velocity estimation, the PRF was adjusted to a higher setting [33]. 
Velocity estimations with conventional US were performed with combined B-mode and SDUS. Aliasing was avoided by adjusting the scale of the spectrogram by increasing the PRF and lowering the baseline. The depicted velocity curve was thus continuous without wrapping. Also, when adjusting the spectral gain as recommended, the flow curve was distinguishable from the background noise [33].

\section{Tools for grading and assessing venous disease}

The included patient population underwent a range of classifications and questionnaires, patient- and physician-based, used for the characterization and severity assessment of chronic venous disease. The Clinical-Etiology-Anatomy-Pathophysiology classification, and Venous Segmental Disease Score were used for characterization by objective measures, while the Visual Analog Scale, Aberdeen Varicose Vein Questionnaire and Venous Clinical Severity Score were used to assess the severity of disease in each patient by subjective measures [34-36]. The former two were used for describing the patient population ( $\triangleright$ Table 1 ), while the latter three were used to correlate disease severity and peak reflux velocities measured with VFI and SDUS. The Visual Analog Scale score represented pain over the last 14 days, and ranged from 0 to 100 [34]. The Aberdeen Varicose Vein Symptom Severity Score ranged from 0 to 100 [35], and the Venous Clinical Severity Score is a 30-point score evaluating 10 items $[1,35,36]$.

Additionally, demographic information and previously performed venous interventions, vessel diameter and vessel depth were obtained ( $\triangleright$ Table $\mathbf{1}$ ).

\section{Statistics}

In the phantom setup, VFI and SDUS measurements were summarized by their bias (mean deviation from truth), relative bias (in \% of true velocity), the absolute precision (standard deviation), and relative precision as measured by the coefficient of variation and error factor. Biases were compared between VFI and SDUS using two-sample t-tests and standard deviations by use of folded F-tests. There is no valid statistical test for comparing two coefficients of variation, and therefore, differences in relative precision were assessed by comparing the error factors (i. e., the standard deviations of log-transformed data). Similar tests were applied to assess differences in performance at the two different velocities for each technique in turn.

In vivo measurements of VFI and SDUS were summarized as the mean, standard deviation and coefficient of variation in each of the 64 patients and compared between the two methods using paired t-tests. Agreement between VFI and SDUS peak reflux velocities was further analyzed in Bland-Altman plots and histograms of absolute and relative differences between VFI and SDUS peak reflux velocities ( $\mathbf{F i g} . \mathbf{2}$ ). The relative difference was calculated as:

Differences of mean in \% $=($ SDUS velocity $-\mathrm{VFI}$ velocity $) /$ mean velocity $* 100$

Linear regression was used for assessing proportional bias in the Bland-Altman plots. The null hypothesis was that there was no proportional bias.

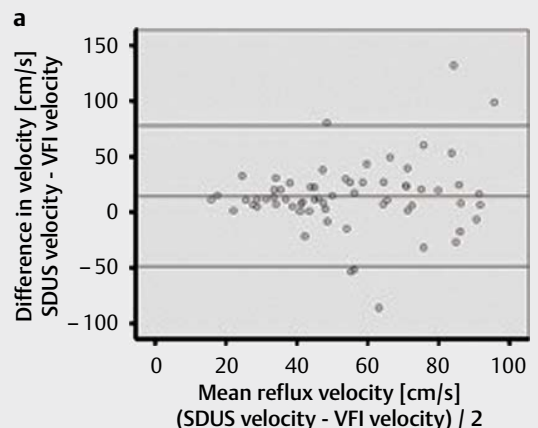

C

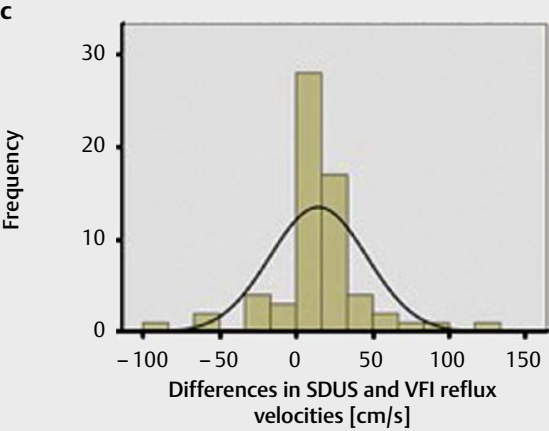

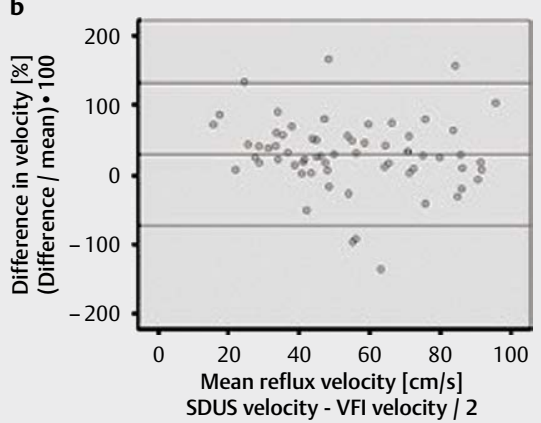

d

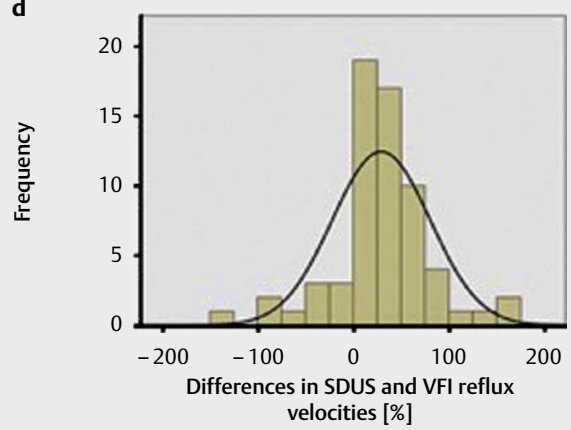

- Fig. 2 a Bland-Altman plot with mean difference of $14.55 \mathrm{~cm} / \mathrm{s}$ and limits of agreement from -48.51 to $77.61 \mathrm{~cm} / \mathrm{s}$. As the scatter has a higher spread toward the right of the figure, at higher mean velocities, the conic shape is a visual sign of increasing variability at increasing mean velocities. b Bland-Altman plot with mean difference of $29.32 \%$ and limits of agreement from -73.24 to $131.88 \%$. The variability in velocity differences at different mean velocities did not occur when depicted relatively using > eq. 1. c Histogram of absolute differences in SDUS and VFI peak reflux velocities with a normally distributed curve superimposed. $\mathbf{d}$ Histogram of relative differences in SDUS and VFI peak reflux velocities with a normally distributed curve superimposed. 
A significance level of 0.05 was chosen. SPSS version 24 (IBM, Armonk, NY, USA) and SAS enterprise guide version 6.1 (SAS Institute, Cary, NC, USA) were used for the statistical analyses.

\section{Results}

\section{Flow phantom}

The bias of VFI and SDUS at $50 \mathrm{~cm} / \mathrm{s}$ did not differ $(p=0.515)$, while a lower bias for VFI compared to SDUS was found at $60 \mathrm{~cm} / \mathrm{s}$ $(p<0.001)(\triangleright$ Table 2). All measurements were above the true velocity, so in all reported cases the bias was identical to the accuracy (mean absolute difference). No significant differences were found in absolute and relative precision between the two techniques at the two velocity settings ( $\triangleright$ Table 2 ). The accuracy of VFI differed for the two velocity settings $(p<0.01)$, as for SDUS $(p<0.001)$. The coefficients of variation of VFI were similar to SDUS at the two velocity settings: $\operatorname{VFI}(50 \mathrm{~cm} / \mathrm{s}: 2.15 \% ; 60 \mathrm{~cm} / \mathrm{s}: 2.11 \%)$ and SDUS (50 cm/s: $3.1 \% ; 60 \mathrm{~cm} / \mathrm{s}: 1.94 \%$ ) (• Table 2).

\section{In vivo}

In the clinical setup, both methods detected pathological retrograde flow for all patients. VFI and SDUS estimated different peak reflux velocities $(p<0.001)$, while no differences in absolute precision $(p=0.177)$ or relative precision $(p=0.789)$ were found ( $\vee$ Table 3$)$. A significant although weak correlation was found between the peak reflux velocities obtained with the two methods $(r=0.28, p=0.023)$. No significant correlations were found between the peak reflux velocities determined by either VFI or SDUS and the tools for assessing chronic venous disease symptom severity (Visual Analog Scale, Aberdeen Varicose Vein Questionnaire, and Venous Clinical Severity Score).

The mean difference in VFI and SDUS peak reflux velocities was $14.55 \mathrm{~cm} / \mathrm{s}$, and the relative mean difference was $29.32 \%$. The absolute and relative velocity differences were depicted as a function of the mean velocity of the two techniques in the Bland-Altman plots ( $\triangleright$ Fig. 2a,b), and corresponding histograms with normal distribution of the data ( $\nabla \mathbf{F i g} . \mathbf{2 c}, \mathbf{d}$ ). The variability in differences seemed to increase with increasing velocities in the Bland-Altman plot of absolute velocities. Hence, limits of agreement were better estimated in relative terms, in which case they ranged from $-73.24 \%$ to $131.88 \%$. It was not possible to reject the null hypothesis of no proportional bias (linear regression of absolute mean: $p=0.263$; linear regression of relative mean: $p=0.270$ ), and no skewness in the data was present in either of the histograms.

\section{Discussion}

The main findings in vitro suggest that VFI performed slightly more accurately than SDUS, while the precision was equal for the two techniques ( $\triangleright$ Table 2 ), as no difference in absolute nor relative precision was found at the two velocity settings. The phantom results are in line with previously published studies and indicate that VFI is comparable to SDUS for the estimation of velocities expected for venous reflux evaluation, even though the measurements in vitro were conducted with a fixed and known angle, which favored SDUS.

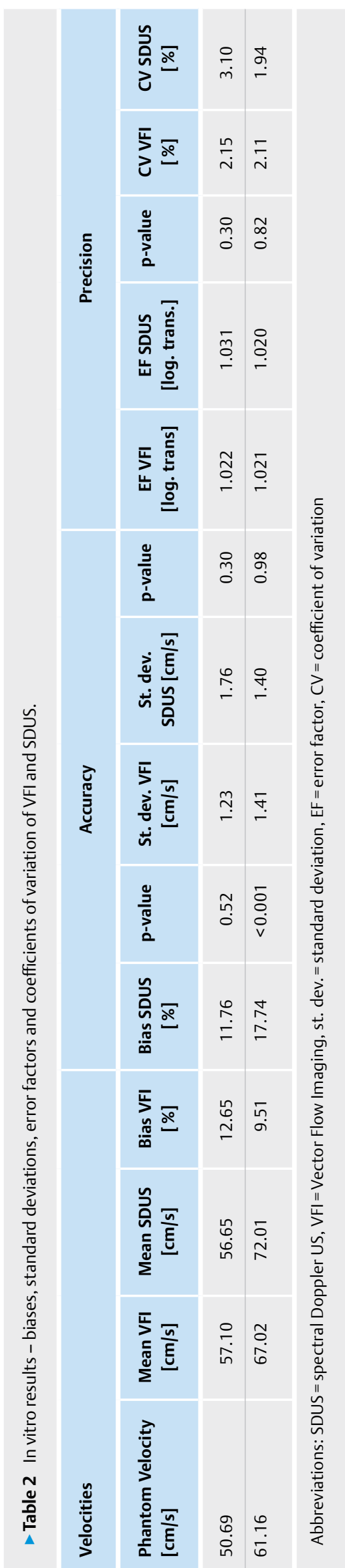


- Table 3 In vivo results - peak reflux velocities, precisions and correlations with clinical scores of VFI and SDUS.

\begin{tabular}{|c|c|c|c|c|c|c|c|c|}
\hline$n=64$ & VFI & SDUS & p-value & & Mean (range) & St. dev. & $\begin{array}{l}\text { Correlation of } \\
\text { VFI (p-value) }\end{array}$ & $\begin{array}{l}\text { Correlation of } \\
\text { SDUS (p-value) }\end{array}$ \\
\hline $\begin{array}{l}\text { Mean PRV } \\
{[\mathrm{cm} / \mathrm{s}]}\end{array}$ & 47.42 & 61.98 & $<0.001$ & $\begin{array}{l}\text { Visual analog scale (pain last } \\
14 \text { days) }\end{array}$ & $29.96(1-78)$ & 21.31 & $0.10(0.43)$ & $0.00(0.97)$ \\
\hline $\begin{array}{l}\text { Mean st. dev. } \\
{[\mathrm{cm} / \mathrm{s}]}\end{array}$ & 6.45 & 9.96 & 0.177 & $\begin{array}{l}\text { Aberdeen varicose vein } \\
\text { questionnaire }\end{array}$ & $12.36(2-37)$ & 6.80 & $0.17(0.19)$ & $0.09(0.48)$ \\
\hline Mean CV [\%] & 14.42 & 13.77 & 0.789 & Venous clinical severity score & $5.15(1-11)$ & 2.36 & $0.09(0.48)$ & $0.09(0.47)$ \\
\hline
\end{tabular}

Abbreviations: VFI = Vector Flow Imaging, SDUS = spectral Doppler ultrasound, st. dev. = standard deviation, $\mathrm{CV}=$ coefficient of variation

The main in vivo findings showed that pathologic retrograde venous blood flow in the great or small saphenous vein could be identified in all patients with both techniques. The VFI and SDUS technique estimated significantly different peak reflux velocities with no differences in absolute or relative precision ( $>$ Table 3 ). VFI and SDUS velocities have previously been compared in different vessels including the deep veins of the lower extremities [21], the portal vein [13], the ascending aorta [37], arteriovenous fistulas [18], the heart [38], and the carotid arteries [15, 26, 39]. In most of these studies, SDUS velocities were above the VFI velocities as in this study. Equal precision of VFI compared to SDUS in vivo has likewise been reported previously for blood flow estimation in e. g. the deep veins of the lower extremities [21], the carotid arteries [39], and the ascending aorta [40].

Increasing bias between VFI and SDUS at increasing peak velocities has been indicated by other papers concerning previous VFI implementation $[21,37,40]$. Hansen et al. reported an increasing bias for increasing velocities, and suggested a correction algorithm for the systematic bias [40]. No systematic bias between the two methods was present for venous reflux estimation ( $\mathbf{F i g}$. $2 \mathbf{b}$ ).

In this study, reduced precision for VFI and SDUS was found in vivo compared to in vitro. This has also been reported for velocity estimations in the flow phantom with constant versus pulsatile flow [41], as for in vitro estimation compared to in vivo [21, 40, 42, 43]. The main reason for the difference in this study is probably that the in vitro flow was constant, while the in vivo blood flow was pulsatile.

Even though the venous reflux in vivo was provoked in the standardized setup, variables influenced the velocity estimates. The instrumental settings of the ultrasound scanner, variation in placement of the transducer on the patient, echo cancelling of moving tissue, and patient specific parameters including cooperation and habitus had an impact on velocity estimates of both methods. Operator-dependent instrumental factors, like placement of range gate, range of velocity scale, Doppler gain, beam steering, and insonation angle were additional concerns in the peak velocity estimation with SDUS [7, 41, 44-47].

Reflux duration is used for diagnosing chronic venous disease in the saphenous veins [1], though no significant discrimination power has been found [5]. Konoeda et al. and Yamaki et al. have suggested peak reflux velocities for discriminating between early to moderate stage chronic venous disease and more advanced chronic venous disease $[4,5]$. In both studies, peak reflux velocities were significantly higher for more advanced disease, and almost identical average peak reflux velocities were reported for the early (C1-C3) and the advanced stage chronic venous disease group (C4C6 in the Clinical-Etiology-Anatomy-Pathophysiology classification) ( $\triangleright$ Table 1). In this present study, the average SDUS peak reflux velocity was higher compared to recently reported average peak reflux velocities, despite the patients almost exclusively presenting with early stage chronic venous disease in this study opposed to the compared studies ( $\triangleright$ Table 1 ). On the contrary, the mean VFI peak reflux velocity of $47.2 \mathrm{~cm} / \mathrm{s}$ was close to the mean SDUS peak reflux velocities of $43.8 \mathrm{~cm} / \mathrm{s}$ reported by Konoeda et al. and of $43.4 \mathrm{~cm} / \mathrm{s}$ reported by Yamaki et al. in the early to moderate stage chronic venous disease group $[4,5]$. The two studies came from the same institution and may have been performed with identical examination techniques, which can explain the high agreement in average peak velocities [41].

In this study, no correlations were found between peak reflux velocities of VFI and SDUS and the three symptom grading systems for chronic venous disease (Visual Analog Scale, Aberdeen Varicose Vein Questionnaire, and Venous Clinical Severity Score). Neither the Clinical-Etiology-Anatomy-Pathophysiology classification nor the Venous Segmental Disease Score was used as most of the patients were identical in these two classifications, that is C2 in the Clinical-Etiology-Anatomy-Pathophysiology classification and single vessel involvement in the Venous Segmental Disease Score ( $\triangleright$ Table 1). The lack of correlation in this study can be explained by the fact that the included patient population was too homogeneous.

In the clinical setting, qualitative flow analysis is used more than quantitative flow estimation, e.g. peak velocity estimation $[2,4,5]$. VFI detected reflux in all patients as did SDUS. It must, however, be underlined that this study did not examine the diagnostic accuracy of the two techniques, as all included patients suffered from chronic venous disease presenting with varicose veins caused by valve incompetence in the great or the small saphenous vein. The direction of blood flow is readily interpreted with VFI ( $>$ Fig. 1). As VFI offers equal precision compared to conventional SDUS, the additional advantages of the technique should be exploited. New insonation windows, improved operator agreement, and applications for quantifying complex flow and volume flow with 3D ultrasound should be examined further [21, 42, 48, 49].

The homogeneous patient population in contrast to previously performed studies with representation from the entire disease spec- 
trum was a limitation in this study [4, 5]. Surgery has previously been performed in $42 \%$ of patients, which may have influenced the results of this study ( Table 1). Measurements with the compared techniques were not obtained simultaneously, which also may have influenced the results. The blood flow velocity of a varicose saphenous vein may vary depending on the distance to the nearest sufficient valve, and the reflux velocity provoked by cuff compression may be related to the body mass index. No inter- and intraobserver variations were examined, and no solid ground truth was available for reference. SDUS is operator- and angle-dependent and is liable to overestimate velocities due to spectral broadening [7,32,44]. Whether VFI or SDUS estimates the most accurate peak reflux velocities in vivo can only be clarified with an independent gold standard, e. g. phase contrast magnetic resonance angiography (MRA) [50]. However, superficial veins are small, and valid results would be a challenge given the limited spatial and temporal resolution of MRA [51].

\section{Conclusion}

The study showed that both VFI and SDUS detect pathological retrograde venous blood flow in all chronic venous disease patients. No significant difference in the precision of peak reflux velocity was found. The mean peak reflux velocity was lower for VFI compared to SDUS, and the correlation between the VFI and SDUS velocity was low. No significant correlations were found between the VFI or SDUS peak reflux velocities and the clinical assessment tools for chronic venous disease. In vitro, VFI was more accurate and equally precise when compared to SDUS for velocity estimation.

Further research will show if additional features of VFI, e.g. estimation of flow complexity and volume flow, can benefit patients with venous disease.

\section{Conflict of Interest}

The Danish National Advanced Technology Foundation and BK Ultrasound supported the project. The author JAJ developed and patented the Transverse Oscillation technique and earns royalties from the sale of scanners with the Transverse Oscillation Vector Flow Imaging technique implemented from BK Ultrasound.

\section{References}

[1] Wittens C, Davies AH, Bækgaard N, Broholm R, Cavezzi A, Chastanet S, de Wolf M, Eggen C, Giannoukas A, Gohel M, Kakkos S, Lawson J, Noppeney T, Onida S, Pittaluga P, Thomis S, Toonder I, Vuylsteke M, Esvs Guidelines C, Kolh P, de Borst G], Chakfe N, Debus S, Hinchliffe R, Koncar I, Lindholt J, de Ceniga MV, Vermassen F, Verzini F, Document R, De Maeseneer MG, Blomgren L, Hartung O, Kalodiki E, Korten E, Lugli M, Naylor R, Nicolini P, Rosales A. Editor's Choice - Management of Chronic Venous Disease: Clinical Practice Guidelines of the European Society for Vascular Surgery (ESVS). Eur J Vasc Endovasc Surg 2015; 49: 678-737

[2] Spinedi L, Broz P, Baldi T, Imfeld S, Staub D, Jaeger K, Aschwanden M, Uthoff H. Evaluation of Varicose Veins of the Lower Extremity: The Value of the Duplex Ultrasound (Part 1). Ultraschall der Medizin 2016; 37: 348-365
[3] Spinedi L, Aschwanden M, Broz P, Imfeld S, Baldi T, Jaeger K, Staub D, Uthoff H, Uthoff H. [Endoluminal Treatment of Varicose Veins: Value of Duplex Ultrasound (Part 2)]. Ultraschall Med 2017; 38: 14-32

[4] Konoeda H, Yamaki T, Hamahata A, Ochi M, Sakurai H. Quantification of Superficial Venous Reflux by Duplex Ultrasound-Role of Reflux Velocity in the Assessment the Clinical Stage of Chronic Venous Insufficiency. Ann Vasc Dis 2014; 7: 376-382

[5] Yamaki T, Nozaki M, Fujiwara O, Yoshida E. Comparative evaluation of duplex-derived parameters in patients with chronic venous insufficiency: Correlation with clinical manifestations. J Am Coll Surg 2002; 195 : 822-830

[6] Chiesa R, Marone EM, Limoni C, Volonte M, Petrini O. Chronic venous disorders: Correlation between visible signs, symptoms, and presence of functional disease. J Vasc Surg 2007; 46: 322-330

[7] Park MY, Jung SE, Young Byun J, Kim JH, Joo GE. Effect of beam-flow angle on velocity measurements in modern Doppler ultrasound systems. AJR Am J Roentgenol 2012; 198: 1139-1143

[8] Jensen JA, Munk P. A new method for estimation of velocity vectors. IEEE Trans Ultrason Ferroelectr Freq Control 1998; 45: 837-851

[9] Møller Pedersen M, Pihl M], Haugaard P, Hansen KL, Lange T, Lönn L, Nielsen MB, Jensen JA. Novel flow quantification of the carotid bulb and the common carotid artery with vector flow ultrasound. Ultrasound Med Biol 2014; 40: 2700-2706

[10] Hansen PM, Olesen JB, Pihl MJ, Lange T, Heerwagen S, Pedersen MM, Rix M, Lönn L, Jensen JA, Nielsen MB. Volume Flow in Arteriovenous Fistulas Using Vector Velocity Ultrasound. Ultrasound Med Biol 2014; 40: $2707-2714$

[11] Hansen KL, Udesen J, Gran F, Jensen JA, Bachmann Nielsen M. In-vivo examples of flow patterns with the fast vector velocity ultrasound method. Ultraschall Med 2009; 30: 471-477

[12] Hansen KL, Møller-Sørensen H, Kjærgaard J, Jensen MB, Lund JT, Pedersen MM, Lange T, Jensen JA, Nielsen MB. Intra-operative Vector Flow Imaging Using Ultrasound of the Ascending Aorta among 40 Patients with Normal, Stenotic and Replaced Aortic Valves. Ultrasound Med Biol 2016; 42: 2412-2422

[13] Brandt AH, Moshavegh R, Hansen KL, Bechsgaard T, Lönn L, Jensen JA, Nielsen MB. Vector flow imaging compared with pulse wave Doppler for estimation of peak velocity in the portal vein. Ultrasound Med Biol 2017; 44: 593-601

[14] Fadnes S, Wigen MS, Nyrnes SA, Lovstakken L. In vivo intracardiac vector velocity imaging using phased array transducers for pediatric cardiology. IEEE Trans Ultrason Ferroelectr Freq Control 2017; 64: 1318-1326

[15] Ekroll IK, Dahl T, Torp H, Løvstakken L. Combined vector velocity and spectral doppler imaging for improved imaging of complex blood flow in the carotid arteries. Ultrasound Med Biol 2014; 40: 1629-1640

[16] Faurie J, Baudet M, Assi KC, Auger D, Gilbert G, Tournoux F, Garcia D. Intracardiac Vortex Dynamics by High-Frame-Rate Doppler Vortography-In Vivo Comparison With Vector Flow Mapping and 4-D Flow MRI. IEEE Trans Ultrason Ferroelectr Freq Control 2017; 64: 424-432

[17] Goddi A, Bortolotto C, Fiorina I, Raciti MV, Fanizza M, Turpini E, Boffelli G, Calliada F. High-frame rate vector flow imaging of the carotid bifurcation. Insights Imaging 2017; 8: 319-328

[18] Fiorina I, Raciti MV, Goddi A, Cantisani V, Bortolotto C, Chu S, Calliada F. Ultrasound Vector Flow Imaging - could be a new tool in evaluation of arteriovenous fistulas for hemodialysis? J Vasc Access 2017; 18: 284-289

[19] Yiu BYS, Lai SSM, Yu ACH. Vector Projectile Imaging: Time-resolved dynamic visualization of complex flow patterns. Ultrasound Med Biol 2014; 40: 2295-2309

[20] Bechsgaard T, Hansen KL, Brandt AH, Holbek S, Lönn L, Strandberg C, Bækgaard N, Nielsen MB, Jensen JA. Blood flow velocity in the popliteal vein using transverse oscillation ultrasound. Proc SPIE Med Imaging 2016; 9790: 1-8 
[21] Bechsgaard T, Hansen KL, Brandt AH, Holbek S, Forman JL, Strandberg C, Lönn L, Bækgaard N, Jensen JA, Nielsen MB. Vector and Doppler ultrasound velocities evaluated in a flow phantom and the femoropopliteal vein. Ultrasound Med Biol 2017; 43: 2477-2487

[22] Dunmire B, Beach KW, Labs KH, Plett M, Strandness DE. Cross-beam vector Doppler ultrasound for angle-independent velocity measurements. Ultrasound Med Biol 2000; 26: 1213-1235

[23] Jensen J, Nikolov S, Yu ACH, Garcia D. Ultrasound Vector Flow Imaging: I: Sequentiel Systems. IEEE Trans Ultrason Ferroelectr Freq Control 2016; 63: 1704-1721

[24] Jensen J, Nikolov S, Yu ACH, Garcia D. Ultrasound Vector Flow Imaging: II: Parallel Systems. IEEE Trans Ultrason Ferroelectr Freq Control 2016; 3010: 1-1

[25] Hansen KL, Nielsen MB, Jensen JA. Vector velocity estimation of blood flow - A new application in medical ultrasound. Ultrasound 2017; 25: 189-199

[26] Goddi A, Fanizza M, Bortolotto C, Raciti MV, Fiorina I, He X, Du Y, Calliada F. Vector flow imaging techniques: An innovative ultrasonographic technique for the study of blood flow. J Clin Ultrasound 2017; 45: 582-588

[27] Au J, Hughson R, Yu A. Riding the plane wave: Considerations for in vivo study designs employing high frame rate ultrasound. Appl Sci 2018; 8: 1-12

[28] Yamaki T, Nozaki M, Sakurai H, Takeuchi M, Soejima K, Kono T. Comparison of manual compression release with distal pneumatic cuff maneuver in the ultrasonic evaluation of superficial venous insufficiency. Eur J Vasc Endovasc Surg 2006; 32: 462-467

[29] van Bemmelen PS, Bedford G, Beach K, Strandness DE. Quantitative segmental evaluation of venous valvular reflux with duplex ultrasound scanning. J Vasc Surg 1989; 10: 425-431

[30] Eberhardt RT, Raffetto JD. Chronic venous insufficiency. Circulation 2014; 130: 333-346

[31] Mitchell DG. Color Doppler imaging: Principles, limitations, and artifacts. Radiology 1990; 177: 1-10

[32] Rubens D], Bhatt S, Nedelka S, Cullinan J. Doppler Artifacts and Pitfalls. Radiol Clin North Am 2006; 44: 805-835

[33] Rumack CM, Levine D. Diagnostic Ultrasound. 5th AuflPhiladelphia: Elsevier; 2018

[34] Cavezzi A, Mosti G, Campana F, Tessari L, Bastiani L, Urso SU. Catheter Foam Sclerotherapy of the Great Saphenous Vein, with Perisaphenous Tumescence Infiltration and Saphenous Irrigation. Eur J Vasc Endovasc Surg 2017; 1-7

[35] Catarinella FS, Nieman FHM, Wittens CHA. An overview of the most commonly used venous quality of life and clinical outcome measurements. J Vasc Surg Venous Lymphat Disord 2015; 3: 333-340

[36] Vasquez MA, Rabe E, McLafferty RB, Shortell CK, Marston WA, Gillespie D, Meissner MH, Rutherford RB. Revision of the venous clinical severity score: Venous outcomes consensus statement: special communication of the American Venous Forum Ad Hoc Outcomes Working Group. J Vasc Surg 2010; 52: 1387-1396

[37] Hansen KL, Møller-Sørensen H, Pedersen MM, Hansen PM, Kjaergaard J, Lund JT, Nilsson JC, Jensen JA, Nielsen MB. First report on intraoperative vector flow imaging of the heart among patients with healthy and diseased aortic valves. Ultrasonics 2015; 56: 243-250
[38] Fadnes S, Nyrnes SA, Torp H, Løvstakken L. Shunt flow evaluation in congenital heart disease based on two-dimensional speckle tracking. Ultrasound Med Biol 2014; 40: 2379-2391

[39] Tortoli P, Lenge M, Righi D, Ciuti G, Liebgott HE, Ricci S. Comparison of carotid artery blood velocity measurements by vector and standard Doppler approaches. Ultrasound Med Biol 2015; 41: 1354-1362

[40] Hansen KL, Møller-Sørensen H, Kjærgaard J, Jensen MB, Lund JT, Pedersen MM, Olesen JB, Jensen JA, Nielsen MB. Vector flow imaging compared with conventional doppler ultrasound and thermodilution for estimation of blood flow in the ascending aorta. Ultrason Imaging 2017; 39: 3-18

[41] Normahani P, Aslam M, Martin G, Standfield N, Jaffer U. Variation in duplex peak systolic velocity measurement in a multi-site vascular service. Perfusion 2015; 30: 636-642

[42] Brandt AH, Hansen KL, Nielsen MB, Jensen JA. Velocity estimation of the main portal vein with Transverse Oscillation. Proc IEEE Ultrason Symp 2015; 1-4

[43] Ricci S, Vilkomerson D, Matera R, Tortoli P. Accurate blood peak velocity estimation using spectral models and vector doppler. IEEE Trans Ultrason Ferroelectr Freq Control 2015; 62: 686-696

[44] Lui EY, Steinman AH, Cobbold RS, Johnston KW. Human factors as a source of error in peak Doppler velocity measurement. J Vasc Surg 2005; 42: 972-979

[45] Steinman AH, Yu AC, Johnston KW, Cobbold RS. Effects of beam steering in pulsed-wave ultrasound velocity estimation. Ultrasound Med Biol 2005; 31: 1073-1082

[46] Steinman AH, Tavakkoli J, Myers JG, Cobbold RSC, Johnston KW. Sources of error in maximum velocity estimation using linear phased-array Doppler systems with steady flow. Ultrasound Med Biol 2001; 27: 655-664

[47] Beach KW, Bergelin RO, Leotta DF, Primozich JF, Sevareid PM, Stutzman ET, Zierler RE. Standardized ultrasound evaluation of carotid stenosis for clinical trials: University of Washington Ultrasound Reading Center. Cardiovasc Ultrasound 2010; 8: 39

[48] Hansen KL, Møller-Sørensen H, Kjærgaard J, Jensen MB, Jensen JA, Nielsen MB. Aortic valve stenosis increases helical flow and flow complexity: A study of intra-operative cardiac vector flow imaging. Ultrasound Med Biol 2017; 43: 1607-1617

[49] Holbek S, Ewertsen C, Bouzari H, Pihl MJ, Hansen KL, Stuart MB, Thomsen C, Nielsen MB, Jensen JA. Ultrasonic 3-D vector flow method for quantitative in vivo peak velocity and flow rate estimation. IEEE Trans Ultrason Ferroelectr Freq Control 2017; 64: 544-554

[50] Jiang J, Strother C, Johnson K, Baker S, Consigny D, Wieben O, Zagzebski J. Comparison of blood velocity measurements between ultrasound Doppler and accelerated phase-contrast MR angiography in small arteries with disturbed flow. Phys Med Biol 2011; 56: 1755-1773

[51] Markl $\mathrm{M}$ et al. Comprehensive 4D velocity mapping of the heart and great vessels by cardiovascular magnetic resonance. J Cardiovasc Magn Reson 2011; 13: 7 